\title{
Mejoramiento genético de sorgo en el INIFAP
}

\author{
Noé Montes-García ${ }^{1 \S}$ \\ Víctor Pecina-Quintero ${ }^{2}$ \\ Héctor Williams-Alanis ${ }^{3}$ \\ Miguel Hernández-Martínez ${ }^{2}$ \\ Ma. Eugenia Cisneros-López ${ }^{1}$ \\ Tomas Moreno-Gallegos ${ }^{4}$
}

${ }^{1}$ Campo Experimental Río Bravo-INIFAP. Carretera Reynosa-Matamoros km 60, Ciudad Río Bravo, Tamaulipas, México. CP. 88900. (cisneros.eugenia@ inifap.gob.mx). ${ }^{2}$ Campo Experimental Bajío-INIFAP. Carretera Celaya-San Miguel de Allende km 6.5, Celaya, Guanajuato. (pecina.victor@inifap.gob.mx; hernández.miguel@inifap.gob.mx). ${ }^{3}$ Ex-Investigador del INIFAP del Campo Experimental Río Bravo. ${ }^{4}$ Campo Experimental Valle de Culiacán-INIFAP. Carretera Culiacán-El Dorado km 17.5, Culiacán, Sinaloa, México. Tel. 6678461014.

${ }^{\S}$ Autor para correspondencia: montes.noe@inifap.gob.mx.

\section{Resumen}

El mejoramiento genético de sorgo en México inició en 1944 en la oficina de Estudios Especiales. Posteriormente, las actividades de mejoramiento se realizaron en tres regiones con la introducción de material genético de donde se derivaron líneas y se generaron los primeros híbridos de sorgo adaptados. El programa se ha enfocado a la generación de material genético para diversos nichos de mercado entre los que sobresalen el de alimento balanceado, alimento para consumo humano y el de producción de biocombustibles. Entre los materiales genéticos sobresale el híbrido RB-3030 por haber sido en su momento el de mayor producción por parte de la PRONASE. A la fecha se han generado 36 híbridos y variedades de los cuales actualmente están siendo aprovechados 12 , dentro de los que sobresalen los sorgos de grano rojo-naranja RB-Norteño y RB-Huasteco y la variedad de sorgo blanco RB-Paloma. Así mismo, sobresalen los sorgos forrajeros Sinaloense 202 y Gavatero-203. Por otra parte, se han formado los sorgos RB-Cañero, RB Cañaveral, RB Tehua y RB-Pirulí para la producción de bioetanol. Además, se cuenta con líneas parentales de híbridos que son adquiridas por empresas para la producción de semilla certificada. Existen varios retos como el de incrementar la producción, pero a corto plazo se requiere incrementar la promoción y mercadotecnia de los materiales genéticos, y a mediano plazo fortalecer las investigaciones en el control de enfermedades, tolerancia a sequía y sales y obtener variedades con mejor calidad nutricional y energética.

Palabras claves: semillas, tolerancia, variedades.

Recibido: enero de 2021

Aceptado: marzo de 2021 


\section{Antecedentes}

A inicios de los años 40's, la Secretaría de Agricultura y Ganadería (SAG) creó la Oficina de Estudios Especiales, dedicada a la investigación sobre cultivos básicos para la alimentación, en la cual laboraban conjuntamente el personal técnico adscrito a la Fundación Rockefeller y personal de la SAG. En México, se iniciaron las primeras investigaciones de sorgo en 1944, y en 1961 se creó el Instituto Nacional de Investigaciones Agrícolas (INIA), donde la estación experimental de Roque, Guanajuato fue la primera en la generación de tecnologías sobre sorgo.

Durante el período 1973-74 se definieron tres áreas estratégicas donde se realizaron investigaciones en mejoramiento genético del sorgo, para lo cual la sede de la región norte fue el Campo Experimental Río Bravo, la de la región centro fue el Campo Experimental Bajío y la región sur el Campo Experimental Uxmal. En todas estas áreas se inició con la introducción y evaluación de germoplasma de sorgo proveniente de varias instituciones entre las que figuraron el International Research Center for the Semi-Arid Tropics (ICRISAT) y la Universidad de Texas A\&M.

\section{Aportaciones del programa de mejoramiento genético}

Como resultado de la investigación realizada en el Campo Experimental Bajío durante el periodo 1968-1975, se liberaron para el norte de Tamaulipas los híbridos INIA Zacapil, INIA Tejón, INIA Maratín, INIA Mayapen, INIA Malinche e INIA Janambre (Betancourt-Vallejo, 1975). En el Campo Experimental de Río Bravo se inició el mejoramiento genético de sorgo en el año de 1973. El principal objetivo del programa fue generar y producir híbridos y variedades de sorgo con adaptación al área del Norte de México con tolerancia a las enfermedades, como el carbón de la panoja [Sporisorium reilianum (Kühn) Langdon y Fullerton].

En 1978 se liberaron los sorgos INIA RB-2000, INIA RB-2010 e INIA RB-2020 (Williams-Alanis y Betancourt-Vallejo, 1979). A partir de 1979 se realizó mejoramiento poblacional con germoplasma introducido de Estados Unidos de América, utilizando la fuente de androesterilidad genética de Coes ms3 (en donde ms3ms3= estéril y MS3MS3 o MS3ms3= fértil) (Williams-Alanis et al., 1995).

En 1982 se introdujo la fuente de androesterilidad génico-citoplásmica A2 a las líneas élite del programa (Rodríguez et al., 1994). En 1980 se liberaron para el área del Bajío mexicano los híbridos BJ83 y BJ84 y los sorgos INIA RB-3030 e INIA RB-3006 para el norte de Tamaulipas y en 1989 se liberó para condiciones de riego el híbrido RB-4000, el cual mostró un potencial de rendimiento superior en 500 a $600 \mathrm{~kg} \mathrm{ha}^{-1}$, a los sorgos comerciales recomendados en esa época.

A partir de 1993, algunos genotipos fértiles fueron transformados en líneas A y B mediante el método de retrocruza (Rodríguez y Torres, 1992). Hasta 1994, se habían seleccionado alrededor de 100 pares de líneas A y B, y 54 líneas R tolerantes a enfermedades. En el programa de mejoramiento genético de sorgo se han evaluado, derivado y liberado nuevas líneas progenitoras de sorgo, utilizando la línea SBA22/SBB22 como progenitor materno.

Se formaron y liberaron los híbridos RB-3006 y el RB-Norteño (Montes et al., 2013a) y con la línea SBA25/SBB25 se formó y liberó el RB-Huasteco (Montes et al., 2013b), los cuales han mostrado buena adaptación al noreste de México. 
En relación con variedades de consumo humano se han liberado sorgos de grano blanco entre los que destacan Blanco-86, Tropical-401, Costeño-201, Perla-101, Mazatlán-16, y RB-Paloma, los cuales presentan características para ser utilizados en la industria de la masa y la tortilla, así como en la industria de la panificación. En cuanto a los genotipos forrajeros, se han desarrollado las variedades Gavatero-203, Sinaloense-202, VCS-Diamante, VCS-Tornasol, VCS-Brillante y Fortuna, los cuales se presentan como materiales de doble propósito en prácticas de conservación de forraje como henificado y ensilaje.

De igual manera, en 2010 se liberó el sorgo dulce RB-Cañero que es el primer material genético de sorgo dulce para producir biomasa y azúcares con fines de obtención de bioetanol en México, y al cual le siguieron los sorgos dulces RB-Pirulí, RB Tehua y RB Cañaveral (Cisneros et al., 2018). Cabe recalcar que todos los materiales genéticos tienen su registro en el Catálogo Nacional de Variedades Vegetales del SNICS y que se tiene el título de obtentor de algunos de los híbridos y variedades recientemente liberados.

\section{Producción y comercialización de semilla}

A principios de los 70's, la PRONASE empezó a producir los híbridos liberados por el entonces INIA, hoy INIFAP. En los 80's se producían en el norte de Tamaulipas $28000 \mathrm{t}$ de semilla de sorgo certificada en 21,000 ha; sin embargo, existían algunos problemas que dificultaban la producción comercial de esta semilla, como, la presencia de esterilidad genética ambiental en las líneas A (hembras), diseminación de malezas de zacate Johnson, mal manejo y cuidado de los lotes de producción, dificultades en el aislamiento y falta de coincidencia en floración en los progenitores.

Algunos de estos problemas se han eliminado a través de la tecnología de producción de semilla que se ha generado, así como en la capacitación y comunicación constante a los productores de semilla. A partir de los 80's y hasta 2002 se produjo semilla de los nuevos híbridos liberados por el INIFAP, los cuales no presentaban problemas en la producción de semilla y fueron producidos en Río Bravo (RB), significando $70 \%$ de los sorgos comercializados por PRONASE en el país. Actualmente, los híbridos y variedades formados por el INIFAP han sido producidos $\mathrm{y}$ comercializados por empresas productoras de semillas y patronatos a nivel nacional.

\section{Retos}

Existen retos, pero el más importante es la adopción de los materiales genéticos, para lo cual es imprescindible de contar con programas de difusión y mercadotecnia de los híbridos y variedades del INIFAP, aunado a la realización de convenios de producción de semilla con organizaciones públicas y privadas para así poder impactar el agro mexicano con materiales genéticos competitivos, de bajo costo, adaptados a las diversas áreas y que sean una alternativa viable a todos los productores para elevar su rentabilidad.

\section{Conclusiones}

A través del tiempo, en el programa de mejoramiento genético de sorgo en el INIFAP, se han generado 36 híbridos y variedades de los cuales sobresalen los sorgos de grano rojo RB-Norteño, RB-Huasteco, RB-3030 y la variedad de sorgo blanco RB-Paloma. Asimismo, los sorgos forrajeros 
Sinaloense 202 y Gavatero-203. Por otra parte, se han formado los sorgos RB-Cañero, RB Cañaveral, RB Tehua y RB-Pirulí para la producción de bioetanol. Se cuenta con líneas parentales que están a disposición para la producción de semilla certificada. A corto plazo se requiere mejorar la calidad nutricional y energética del grano y forraje.

\section{Literatura citada}

Betancourt-Vallejo, A. 1975. Resumen de los trabajos de maíz y sorgo del CIAT. In: II Reunión Nacional de Investigadores de maíz y sorgo. INIA. 26-27 de julio. Cotaxtla, Veracruz. $104 \mathrm{p}$.

Cisneros, L. M. E.; Montes, G. N.; Aguilar, U. M. G.; Uribe, G. S. y Rebolledo, G. R. L. 2018. Sorgos dulces para la producción de biomasa y azúcares: RB-Piruli, RB Cañaveral y RB Tehua. INIFAP-CIRNE-CERIB. Folleto técnico núm. 71. 49 p.

Montes, G. N.; Williams, A. H.; Pecina, Q. V.; Arcos, C. G.; Vargas, V. E.; Espinosa, R. M. y Herrera, C. C. 2013a. RB-Norteño: Sorgo granífero para las áreas de temporal de Tamaulipas y Guanajuato. SAGARPA-INIFAP-CIRNE-CERIB. Folleto técnico núm. 55. $32 \mathrm{p}$.

Montes, G. N.; Williams, A. H.; Arcos, C. G.; Pecina, Q. V.; De la Garza, C. M.; Cisneros, L. M. E.; Vargas, V. E. y Herrera, C. C. 2013b. RB-Huasteco: sorgo de grano para elevar la sustentabilidad de las áreas de riego y buen temporal de Tamaulipas y Guanajuato. SAGARPA-INIFAP-CIRNE-CERIB. Folleto técnico núm. 52.35 p.

Rodríguez, H. R. y Torres, M. H. 1992. Esterilización génico-citoplásmica de una línea restauradora de sorgo. I. Segregación y efecto ambiental en el sistema Milo- Kafir (A1). In: III Congreso Nacional de la Sociedad Mexicana de Genética. Mazátlan, Sinaloa. 212$213 \mathrm{pp}$.

Rodríguez, H. R.; Williams, A. H.; Torres, M. H. y Montes, G. N. 1994. Introducción de androesterilidad a una línea restauradora de sorgo. III. Segregación de generaciones avanzadas. In: Memoria XV Congreso Nacional de Fitogenética. 25-30 de septiembre. FAUANL. Marín, Nuevo León. 497 p.

Williams-Alanis, H. y Betancourt-Vallejo, A. 1979. Mejoramiento del sorgo para grano en la región norte de Tamaulipas mediante híbridos provenientes del cruzamiento de líneas A y $\mathrm{R}$ mexicanas (INIA) por introducidas, EUA. In: $\mathrm{X}$ Reunión de la Asociación Latinoamericana de Ciencias Agrícolas. 22 al 28 de abril. Acapulco, Guerrero. 72 p.

Williams-Alanís, H.; Rodríguez-Herrera, R. y Montes-García, N. 1995. 20 años de investigación en sorgo en el Campo Experimental Río Bravo. Germen-Boletín de Intercambio Técnico y Científico de la Sociedad Mexicana de Fitogenética. 11:1-35. 